\title{
Acknowledgment of Reviewers
}

The Editor thanks the following individuals for their assistance in reviewing materials submitted to The Psychologist-Manager Journal in the past year. Their generosity, expertise, and professionalism are very much appreciated.

-Rosemary Hays-Thomas, Editor

Kenneth Ball

John M. Cornwell

Donna Chrobot-Mason

Maria U. Darcy

Claudia Flowers

Leslie B. Hammer
Al Hollenbeck

Paul Lloyd

Rodney L. Lowman

Rebecca Magerkorth Stephen J. Vodanovich

Frada Mozenter

Edward J. Pavur
William D. Siegfried, Jr.

Margaret Stockdale

Marion A. Terenzio 